\title{
Utilization of paediatric isolation facilities in a TB-endemic setting
}

\author{
Angela Dramowski ${ }^{1 *}$, Mark F. Cotton ${ }^{1}$ and Andrew Whitelaw ${ }^{2}$
}

\begin{abstract}
Introduction: In hospital settings, patient isolation is used to limit transmission of certain pathogens (e.g. M. tuberculosis [TB], antibiotic-resistant bacteria and viruses causing respiratory and enteric infection). Data is lacking on utilization of paediatric isolation facilities in low-resource, TB-endemic settings.

Methods: Prospective weekday observation of 18 paediatric isolation rooms at Tygerberg Children's Hospital, Cape Town, South Africa, was conducted between 1 May 2014 and 31 October 2014 documenting: occupancy rate; indication for isolation; duration of isolation; application of transmission-based precautions and infection prevention (IPC) behaviour of personnel. Potential under-utilization of isolation rooms was determined by cross-referencing isolation room occupancy with laboratory isolates of antibiotic-resistant bacteria, M. tuberculosis and selected viral pathogens.

Results: Six percent (335/5906) of hospitalized children were isolated: 78 \% (260/335) for IPC purposes. Most IPC-isolated patients had community-acquired infections (213/260; $82 \%)$, including tuberculosis (130/260; $50 \%$ ) and suspected viral infections (75/260; $29 \%$ ). Children (median age 17 months [IQR 6-50]) spent 4 days (IQR 2-8) in isolation. Isolation occupancy was $66 \%$ (2172/3294 occupied bed days), but varied significantly by month. Laboratory data identified an additional 135 patients warranting isolation with 2054 extra bed-days required. Forty patients with 171 patient days of inappropriate isolation were identified. During 1223 weekday visits to IPC-isolated patient rooms: alcohol-based handrub was available (89\%); transmission-based precautions were appropriately implemented (71 \%); and personal protective equipment was provided (74\%). Of 358 observed interactions between paediatric staff and isolated patients, hand hygiene compliance was $65 \%$ and adherence to transmission-based precautions was $58 \%$.
\end{abstract}

Conclusion: Patients isolated for TB (under airborne precautions) accounted for more than half of all isolation episodes. Missed opportunities for patient isolation were common but could be reduced by implementation of syndromic isolation. Demand for isolation facilities was seasonal, with projected demand exceeding available isolation beds over winter months.

Keywords: Paediatrics, Healthcare-associated infection, Nosocomial infection, Infection control, Patient isolation, Transmission-based precautions, Tuberculosis

\section{Background}

Standard and transmission-based precautions (contact, droplet and airborne) [1] are used to interrupt pathogen transmission in healthcare settings. Patient isolation is a key component of these precautions, targeting pathogens such as M. tuberculosis (TB), antibiotic-resistant bacteria and certain viruses. Despite widespread implementation of these precautions in high-income countries, the resources

\footnotetext{
* Correspondence: dramowski@sun.ac.za

'Department of Paediatrics and Child Health, Division of Paediatric Infectious Diseases, Faculty of Medicine and Health Sciences, Stellenbosch University, PO Box 241, Cape Town 8000, South Africa

Full list of author information is available at the end of the article
}

to effectively apply patient isolation and transmissionbased precaution recommendations are lacking in many low and middle income countries $[2,3]$.

In paediatric wards, where infectious disease relatedadmissions predominate, the demand for isolation beds may be ten-fold greater than for hospitalized adults [4]. Not only are hospitalized children more likely to transmit infection, they are also at elevated risk for healthcareassociated infection (HAI) owing to immunological immaturity, underdeveloped mucosal barriers and increased handling by healthcare staff [5]. Studies of isolation facility usage in high-income settings report that 5-17\% of 
paediatric patients need isolation for IPC purposes, mostly for community-acquired infections (60-75\%) [4, 6-8]. Marked seasonality in demand for paediatric isolation beds (with demand often exceeding supply) is reported even from facilities with more single rooms than cohort beds [4, $6,7]$. The most common precaution type implemented in paediatric studies $[5,8,9]$ is contact precautions (80-90\%) followed by droplet precautions [6]. A single study [6] measured correct use of isolation room precaution signage $(93 \%)$ and availability of personal protective equipment (100\%). Staff compliance with hand hygiene and transmission-based precautions recommendations in paediatric isolation rooms was not evaluated in these studies.

The impact of absent or limited paediatric isolation facilities in low-middle income settings is unquantified, but undoubtedly promotes infection transmission, along with adverse health system factors like overcrowding and lack of IPC provisions [3]. Data on rates of patient isolation, indications for and utilization patterns of paediatric isolation facilities in Africa is lacking. We evaluated isolation facility utilization and transmissionbased precaution implementation at a paediatric referral hospital in a TB-endemic setting in Cape Town, South Africa.

\section{Methods}

\section{Setting and patient profile}

The Tygerberg Children's Hospital (TCH) in Cape Town, South Africa has 300 paediatric beds within the 1384bed academic hospital complex. Sick neonates, infants and children (0-14 years) from Cape Town's Metropole East are hospitalized in 13 neonatal and paediatric wards (including surgical, general medical, sub-specialty wards and intensive care). Admissions reflect a high burden of infectious disease with TB, lower respiratory tract infections and gastroenteritis predominating. HIV prevalence in paediatric inpatients is approximately $15 \%$; antiretroviral therapy is widely available and improved access to prevention of mother-to-child HIV infection transmission programmes (PMTCT) has reduced national vertical HIV transmission rates to $2.4 \%$ in 2012 [9]. Immunisation coverage rates were $88 \%$ in infants under 12 months of age in 2012 [10].

Patient isolation in our context implies placement of a patient in a single room with application of transmissionbased precautions based on clinical indication. Five paediatric wards have 18 single rooms available for patient isolation (9 under negative pressure; only 3 with en-suite bathrooms; none have ante-rooms). This represents $14 \%$ of the available beds on the five wards: 1 room in acute admissions, 3 in paediatric surgery, 2 in general paediatrics, 2 in pulmonology/neurology and 10 in the infectious diseases/gastroenterology ward. The paediatric intensive care unit (PICU) has 10 beds in 2 cohort rooms with no single room or isolation facilities available. PICU patients were eligible for study inclusion if transferred to any of the specified wards.

Each ward's medical and nursing personnel determine which patients are placed in isolation. Although there is no formal policy guiding isolation room utilization, preference is given to patients requiring airborne precautions e.g. pulmonary $\mathrm{TB}$, measles and varicella. Where possible, children are accompanied by a caregiver (who may also require isolation e.g. for pulmonary $\mathrm{TB}$ ). Syndromic isolation for suspected viral diseases is infrequently implemented, owing to clinician unfamiliarity with the practice and limited isolation space. In contrast, syndromic isolation for suspected TB is actively practiced, based on compatible symptoms and signs, history of TB contact and/or a suggestive chest radiograph appearance. The hospital's Unit for Infection Prevention and Control (IPC) conducts laboratory surveillance for selected bacterial "alert" pathogens and makes recommendations for patient isolation on an ad-hoc basis. Infectious patients are usually isolated until discharge (with the exception of meningococcal disease with de-isolation after $24 \mathrm{~h}$ of therapy).

\section{Study design and data collection}

Prospective observation of paediatric isolation rooms was conducted on weekdays from 1 May 2014 to 31 October 2014 documenting: occupancy rates; indication for isolation; duration of patient isolation and application of transmission-based precautions using the 2007 CDC patient isolation indications [1]. During weekday visits, observed interactions between personnel and isolated patients were documented including compliance with hand hygiene and transmission-based precautions. Hand hygiene was scored as compliant if all potential opportunities for hand hygiene were followed, or non-compliant if all or some opportunities for hand hygiene were missed. Transmission-based precautions were scored as compliant if all recommendations and appropriate personal protective equipment was applied, or non-compliant if all or some recommendations were not followed. Alcohol handrub and personal protective equipment was scored as available, if the handrub and equipment items needed (based on the appropriate precautions) were supplied at the entrance to the isolation room.

\section{Estimation of isolation room under-utilization}

Two datasets were created: "patient isolation room utilization data" was collected on weekday ward rounds using Microsoft Access 2013 and "patients with pathogens warranting isolation" was extracted from Microbiology and Virology laboratory databases. Pathogens warranting isolation [1] included: multidrug-resistant (MDR) bacteria [11] from any clinical specimen (blood culture, urine, 
tissue, pus, wound swab and catheter tip) including methicillin-resistant S. aureus [MRSA], carbapenemresistant $A$. baumanni [CRAB], MDR $P$. aeruginosa [MDR PA] and extended spectrum B-lactamase producing Enterobacteriaceae [ESBL]; M. tuberculosis both drug-susceptible (DS) and drug-resistant (DR) isolated from any respiratory sample (gastric washing and/or induced sputum) on GeneXpert, smear microscopy or TB culture; and viruses including enteric (rota and adenovirus, hepatitis A virus) and respiratory pathogens (respiratory syncytial virus, adenovirus, human rhinovirus, parainfluenza $1 / 2 / 3$, influenza $A / B$ and human metapneumovirus) identified by rapid assays, enzyme-linked immunosorbent assays (ELISA) or polymerase chain reaction (PCR) panel testing. All laboratory investigations were taken at the discretion of attending clinicians. Repeated isolates of the same pathogen from one or more sites counted as a single infection episode warranting patient isolation.

The laboratory pathogen dataset was cross-referenced against the patient isolation room utilization dataset. Several measures of isolation room utilization were calculated: missed isolation (patients with pathogens warranting isolation who were not isolated), delayed isolation (patients with late imposition of isolation precautions), inappropriate isolation (no clinical indication for isolation; failure to deisolate after no pathogens or pathogens not warranting isolation were identified and failure to de-isolate after appropriate therapy) and syndromic isolation requirement (patients with suspected viral infections who were not isolated and had a negative laboratory test results for viral pathogens by day four of hospitalisation). Additional isolation bed days required were calculated by adding the length of stay for each patient who was identified as having a missed indication for IPC-isolation. Additional syndromic bed-days were calculated by adding the number of days from hospital admission to a negative test result for specified viral pathogens for each patient (mean interval was 3.5 days). Additional days of pathogen exposure were calculated as days of missed isolation plus days of delayed isolation.

\section{Statistical analysis and ethical approval}

Descriptive statistical analyses were performed using Stata Statistical Software version 13.0 IC (College Station, TX: StataCorp LP). Median length of stay was compared using the Mann-Whitney test. A p-value below 0.05 was considered statistically significant. Ethical approval and waiver of individual informed consent was obtained from the Human Health Research Ethics committee of Stellenbosch University (S13/09/171).

\section{Results}

Six percent $(335 / 5906)$ of children admitted during the study period were placed in isolation. Most isolation episodes (260/335; 78 \%) were for IPC indications. Community-acquired infections were the predominant reason for isolation (213/260; $82 \%)$, including TB disease (130/260; $50 \%)$ and suspected viral infections (75/ 260; $29 \%$ ) (Table 1). Non-IPC indications for isolation included nursing considerations (post-operative care or

Table 1 Paediatric isolation room utilization

\begin{tabular}{|c|c|c|c|}
\hline Variable & Total & Percentage & Interquartile range \\
\hline Discrete patient isolation episodes & 335 & 100 & - \\
\hline Median patient age (months) & 17 & - & $6-50$ \\
\hline Median stay in isolation room (days) & 4 & - & $2-8$ \\
\hline \multicolumn{4}{|l|}{ Indication for isolation } \\
\hline - infection control (IPC) purposes & 260 & 78 & \\
\hline - nursing care & 46 & 14 & - \\
\hline - palliation/privacy & 13 & 4 & \\
\hline - other ${ }^{a}$ & 16 & 4 & \\
\hline Transmission-based precautions ${ }^{b}$ applied & 260 & 100 & \\
\hline - airborne precautions & 136 & 52 & - \\
\hline - droplet precautions & 57 & 22 & \\
\hline \multirow[t]{2}{*}{ - contact precautions } & 67 & 26 & \\
\hline & Mean & Minimum & Maximum \\
\hline \multirow[t]{2}{*}{ Isolation room occupancy rate ${ }^{c}$} & $2172 / 3294$ & $225 / 540$ & $487 / 558$ \\
\hline & $(66 \%)$ & (42\%) & (87\%) \\
\hline
\end{tabular}

IPC = infection prevention and control

a other = no obvious reason for isolation $(n=11)$, behavioural isolation $(n=3)$, protective isolation $(n=2)$

busing the 2007 CDC isolation guidelines[1]

${ }^{\mathrm{C}}$ Calculated as the sum of days when isolation rooms $(n=18)$ were occupied divided by [total isolation bed capacity $\mathrm{x}$ number of days in the observation period] i.e. 6 months to calculate mean or 1 month to calculate minimum and maximum occupancy rates 
provision of total parenteral nutrition), palliation, behavioral or protective isolation. In 11/335 (3\%) episodes, no indication for isolation was identified. Children (median age 17 months; IQR 6-50) were isolated for a median of 4 (IQR 2-8) days. Patients isolated for suspected or confirmed TB stayed longer than patients with other infectious indications for isolation (median of 5 versus 3.5 days; $p=0.006$ ). Overall isolation room occupancy was $66 \%$ (2172 occupied/3294 available bed days), but varied significantly by month with peak usage in winter months (June [76 \%], July [77 \%], August [87\%]).

TB disease (130/260; $50 \%)$ was the most frequent admission diagnosis in IPC-isolated patients [Table 2]. In 55 children $(42 \%)$ microbiological confirmation was obtained; twelve children (9\%) had smear-positive tuberculosis (1-99 acid-fast bacilli per high power field) and 12/45 (26 \%) children with culture-positive TB had drugresistant disease.

The remaining 130/260 (50 \%) patients isolated for IPC indications included suspected or confirmed: viral respiratory infection $(49 ; 19 \%)$, viral gastrointestinal infection (20; $8 \%$ ), varicella or measles $(6 ; 2.5 \%)$, nosocomial sepsis $(29 ; 11 \%)$, skin/soft tissue infection (13; $5 \%)$, hepatitis A (7; $3 \%)$ and meningococcal infection (6; $2.5 \%)$. In some patients (isolated on clinical suspicion of infection), laboratory tests revealed no pathogen or a pathogen not requiring isolation/transmission-based precautions (Table 2).

Cross-referencing of patient isolation and laboratory data identified an additional 135 patients warranting isolation (i.e. missed isolation episodes), with an additional 2054 required isolation bed-days. Of patients with missed isolation episodes, 43 (32\%) had MDR bacteria [1167 extra bed-days], 16 (12\%) had newly-diagnosed, microbiologically confirmed drug-susceptible TB [116 extra bed-days] and 76 (56 \%) had viral pathogens [771 extra bed-days] (Table 3).

Of 395 laboratory investigations for viral pathogens warranting isolation: 103 patients with a positive test were identified ( $26 \%$ yield). Of these 103 patients, 19 (18\%) had been isolated from admission, 8 (8\%) had delayed isolation (mean of 6 days delay) and $76(74 \%)$ were never isolated. The eight delayed isolation episodes had a combined 51 days of additional pathogen exposure on the ward (including hepatitis A, adenovirus, respiratory syncytial virus, parainfluenza 3 and rhinovirus.) A further 226 patients (with 292 negative laboratory tests for viral pathogens warranting isolation) would have qualified for syndromic isolation on admission. Implementing syndromic isolation for these patients would add an additional 990 days of isolation bed demand, increasing the months where demand would outstrip bed availability from 3 to 5 months in the study period (Fig. 1).
Forty patients with 171 patient days of inappropriate isolation were identified (29 days with no apparent reason for isolation and 142 days where IPC isolation was unwarranted i.e. no pathogens were isolated by day 4 of admission). Overall inappropriate isolation days accounted for $5 \%$ of the available bed days (171/3294). Although total projected isolation bed-days reflected a deficit of 761 days (or $123 \%$ projected occupancy) for missed isolation episodes, the deficit was confined to the first 3 months of the study period during the winter season (Fig. 1). The percentage of paediatric admissions requiring isolation increases from $6 \%$ to $8 \%$ when patients with missed isolation episodes are included and to $12 \%$ when including missed isolation and children investigated for viral pathogens.

During 1223 weekday visits to isolation rooms used for IPC: alcohol-based handrub was generally available (89\%); transmission-based precautions were appropriately implemented $(71 \%)$ and personal protective equipment was provided (74\%). Of 358 observed interactions between personnel and isolated patients, hand hygiene compliance was $65 \%$ and adherence to transmissionbased precautions was $58 \%$.

\section{Discussion}

In our study of paediatric isolation room utilization, only $6 \%$ of hospitalized children were placed in isolation. This contrasts with reported isolation rates of 14-17\% in high-income countries, where more single rooms and favourable staffing ratios facilitate greater use of isolation precautions. However, even in high income countries, isolation facilities may be under-utilised. Failure to implement syndromic isolation for suspected viral infections may also explain our institution's lower isolation rates, supported by the finding of many missed isolation opportunities (particularly among children investigated for viral respiratory and enteric infections). Another explanation for our apparently "low" isolation room occupancy and many "missed" episodes, may be a mismatch between theoretical availability of isolation beds and actual availability of beds. At times of peak isolation demand, even with overall $66 \%$ occupancy, clinicians may struggle to find an open isolation bed at the time that the patient requires it.

In keeping with published reports, community-acquired infections predominated, although TB was the most frequent diagnosis among isolated patients at our institution. Consequently airborne precautions were the predominant precaution type implemented, in stark contrast to studies from high-income settings [4,6-8]. Since our institution is a referral centre for complicated and/or drug-resistant $\mathrm{TB}$, the findings may over-estimate requirement for airborne isolation facilities in other low-middle income paediatric settings. However, South African TB incidence 
Table 2 Microbiological isolates ${ }^{\text {a }}$ from patients in isolation for IPC purposes

\begin{tabular}{|c|c|c|}
\hline Category & Variable & Total (\%) \\
\hline \multirow[t]{2}{*}{ All suspected TB $(n=130)$} & pulmonary TB & $118(91)$ \\
\hline & extra-pulmonary $\mathrm{TB}^{\mathrm{b}}$ & $12(9)$ \\
\hline \multirow[t]{4}{*}{ TB smear microscopy $(n=130)$} & not tested (diagnosis confirmed at referral hospital) & $10(8)$ \\
\hline & smear-negative & $108(83)$ \\
\hline & smear-positive (1-10 AFB/field) & $8(6)$ \\
\hline & smear-positive (11-99 AFB/field) & $4(3)$ \\
\hline \multirow[t]{3}{*}{ Confirmed TB $(n=55)$} & TB diagnosis confirmed at referral hospital & 10 \\
\hline & GeneXpert positive & 43 \\
\hline & TB culture positive & 45 \\
\hline \multirow[t]{5}{*}{ Drug-susceptibility profile of culture positive cases $(n=45)$} & Drug-susceptible (DS) & $33(74)$ \\
\hline & Multidrug-resistant (MDR) & $9(20)$ \\
\hline & Rifampicin mono-resistant (RMR) & $2(4)$ \\
\hline & Extensively drug-resistant (XDR) & $1(2)$ \\
\hline & & Total (number DR) \\
\hline \multirow{15}{*}{$\begin{array}{l}\text { All abacterial/fungal isolates cultured from } \\
\text { patients in isolation }(n=46)\end{array}$} & Gram positives & \\
\hline & S. aureus total (methicillin-resistant) & $7(2)$ \\
\hline & Other gram positives $^{d}$ & 4 \\
\hline & Gram negatives: Enterobacteriaceae & \\
\hline & K. pneumoniae (extended-spectrum B-lactamase) & $11(10)$ \\
\hline & E. coli (extended-spectrum B-lactamase) & $5(1)$ \\
\hline & E. cloacae (inducible B-lactamase) & $4(2)$ \\
\hline & Other Enterobacteriaceae ${ }^{e}$ & 4 \\
\hline & Gram negatives: Non-fermenters & \\
\hline & A. baumanni (multi-drug resistant) & $4(4)$ \\
\hline & P. aeruginosa (multi-drug resistant) & $5(3)$ \\
\hline & Others & \\
\hline & N. meningitidis & 2 \\
\hline & B. pertussis (PCR positive) & 1 \\
\hline & C. albicans & 2 \\
\hline \multirow{10}{*}{$\begin{array}{l}\text { Viral pathogens confirmed among patients } \\
\text { in isolation }(n=51)\end{array}$} & Gastrointestinal viruses & \\
\hline & Hepatitis A & 6 \\
\hline & Rotavirus & 4 \\
\hline & Enteric adenovirus & 2 \\
\hline & Respiratory viruses & \\
\hline & Respiratory syncytial virus & 15 \\
\hline & Adenovirus & 12 \\
\hline & Rhinovirus & 4 \\
\hline & Parainfluenza virus & 2 \\
\hline & Other respiratory viruses ${ }^{f}$ & 3 \\
\hline
\end{tabular}


Table 2 Microbiological isolates ${ }^{a}$ from patients in isolation for IPC purposes (Continued)

\begin{tabular}{lll}
\hline & Other & 2 \\
2 \\
Varicella & Rubella & 1 \\
\hline
\end{tabular}

${ }^{\mathrm{a}}$ Some patients were isolated on clinical suspicion of sepsis or suspicion of a pathogen warranting isolation, but laboratory tests subsequently confirmed a pathogen which did not warrant isolation or transmission-based precautions by CDC guidelines[1] e.g. Candida albicans; TB = tuberculosis

${ }^{b}$ Extra-pulmonary TB: disseminated (7), TB lymphadenitis (3), TB meningitis (2)

${ }^{c}$ confirmed $\mathrm{TB}=$ geneXpert positive or culture positive for $\mathrm{mTB}$; $\mathrm{DR}=$ drug-resistant

${ }^{d}$ Other gram positives: C. difficile (1), S. pyogenes (1), S. pneumoniae (1), E. faecium (1)

e Other Enterobacteriaceae: S. typhi (1), S. non-typhi (1), C. freundii (1), P. mirabilis (1)

Other respiratory viruses ${ }^{f}$ : Influenza virus (1), Bocavirus (1), Human metapneumovirus (1)

rates are among the highest worldwide (at 860 per 100000 population) [12] and therefore a strong argument could be made for provision of airborne isolation at all inpatient facilities.

Paediatric TB is often considered low-risk for nosocomial transmission, but our cohort of 130 patients had substantial rates of smear-positive and/or drug-resistant disease. Children with TB also stayed significantly longer (increasing TB exposure time on the wards), despite expedited transfer to a regional TB treatment facility. Another consideration in TB-endemic settings is the presence of undiagnosed or recently diagnosed pulmonary TB in caregivers accompanying hospitalized children, with several documented instances of nosocomial TB transmission [13-17]. Paediatric wards in TB-endemic settings should implement routine symptom screening of adult caregivers and make allowance for additional isolation space for infectious adults, whenever possible.

Most isolation episodes were for IPC purposes, although nursing considerations also influenced isolation room use. Five percent of isolation bed-days were inappropriately used, highlighting the need for a written guideline on

Table 3 Missed opportunities ${ }^{a}$ for patient isolation (May - October 2014)

\begin{tabular}{|c|c|c|c|c|c|c|c|}
\hline \multirow[t]{2}{*}{ Pathogen type and species } & \multicolumn{7}{|c|}{ Missed isolation (Non-isolated patients with pathogens warranting isolation) } \\
\hline & May & June & July & August & Sept. & Oct. & Study period \\
\hline \multicolumn{8}{|l|}{${ }^{\mathrm{b}} \mathrm{MDR}$ bacteria } \\
\hline Methicillin-resistant S. aureus (MRSA) & 3 & 0 & 0 & 1 & 2 & 1 & 7 \\
\hline Carbapenem-resistant $A$. baumanni (CRAB) & 0 & 1 & 0 & 0 & 0 & 2 & 3 \\
\hline MDR P. aeruginosa (MDR PA) & 0 & 0 & 0 & 1 & 2 & 1 & 4 \\
\hline Extended spectrum B-lactamase producing (ESBL) Enterobacteriaceae & 8 & 8 & 5 & 4 & 1 & 3 & 29 \\
\hline \multicolumn{8}{|l|}{${ }^{\mathrm{c}} M$. tuberculosis } \\
\hline Drug-susceptible (DS) & 2 & 0 & 3 & 3 & 3 & 5 & 16 \\
\hline Drug-resistant (DR) & 0 & 0 & 0 & 0 & 0 & 0 & 0 \\
\hline \multicolumn{8}{|l|}{ Enteric viruses } \\
\hline Hepatitis A & 3 & 4 & 5 & 7 & 2 & 2 & 23 \\
\hline Rotavirus and/or enteric Adenovirus & 0 & 2 & 2 & 1 & 0 & 0 & 5 \\
\hline \multicolumn{8}{|l|}{ Respiratory viruses } \\
\hline Respiratory syncytial virus $A / B$ & 9 & 5 & 2 & 2 & 0 & 1 & 19 \\
\hline Adenovirus & 2 & 2 & 3 & 3 & 3 & 1 & 14 \\
\hline${ }^{\mathrm{d}}$ Other respiratory viruses & 0 & 3 & 4 & 2 & 2 & 4 & 15 \\
\hline Missed patient isolation episodes ${ }^{a}$ & 27 & 25 & 24 & 24 & 15 & 20 & 135 \\
\hline Inappropriate isolation room use (days) ${ }^{\mathrm{e}}$ & 29 & 77 & 7 & 30 & 27 & 1 & 171 \\
\hline
\end{tabular}

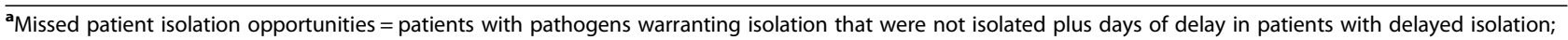
multiple laboratory isolates of the same pathogen from 1 /more sites was considered a single infection episode warranting patient isolation

${ }^{\mathrm{b}}$ Multidrug-resistant (MDR) bacteria isolated from a clinical specimen (blood culture, urine, tissue, pus, wound swab, catheter tip) as per proposed definitions [12] (including methicillin-resistant S. aureus [MRSA], carbapenem-resistant A. baumanni [CRAB], MDR P. aeruginosa [MDR PA] and extended spectrum B-lactamase producing Enterobacteriaceae [ESBL])

${ }^{c} M$. tuberculosis (M.tb) = any form of drug-susceptible (DS) or drug-resistant (DR) M.tb isolated on GeneXpert, smear microscopy or TB culture; Viruses included gastrointestinal and respiratory pathogens identified by rapid assays, ELISA or PCR panels

dOther respiratory viruses warranting isolation and transmission-based precautions = human rhinovirus, parainfluenza 1/2/3, Influenza A/B and

human metapneumovirus

enappropriate isolation room use = total days when isolation rooms were used for inappropriate purposes i.e. without a clinical indication, failure to de-isolate after laboratory testing identified no pathogens or pathogens that did not warrant isolation 


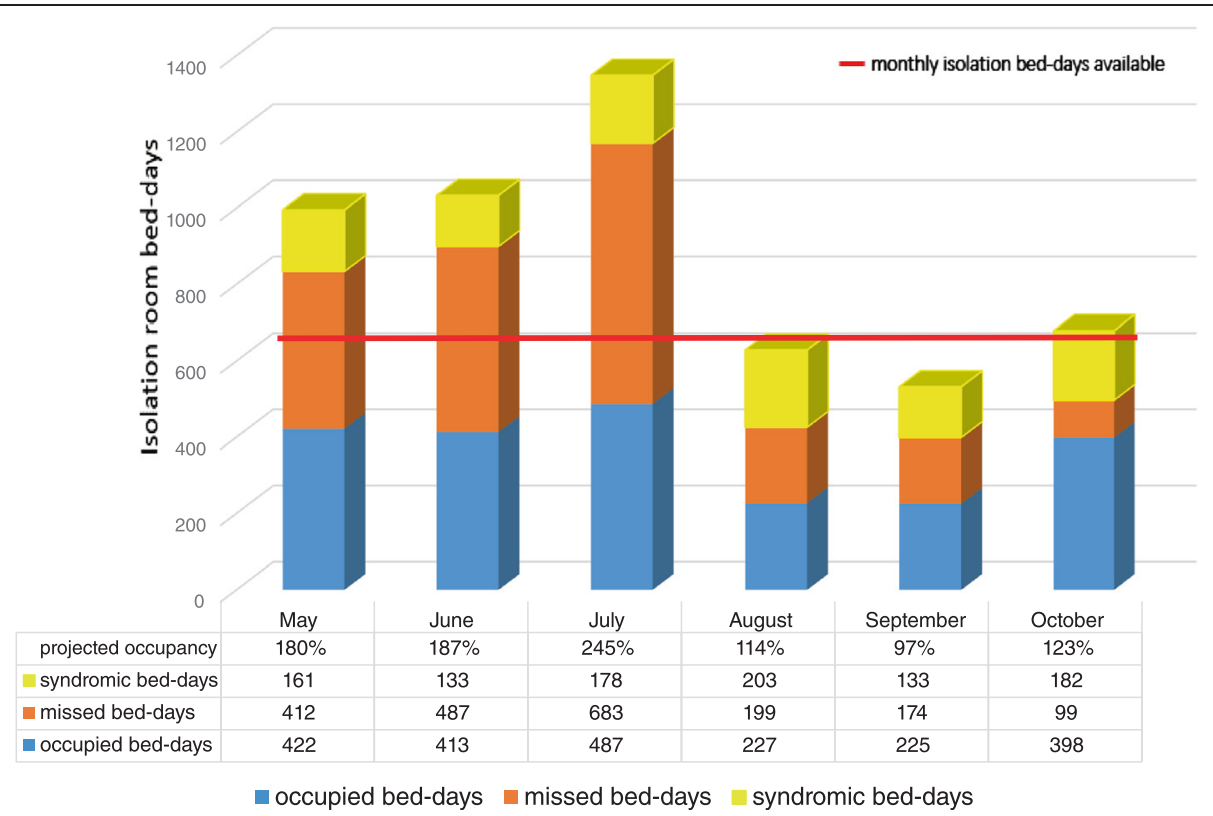

Fig. 1 Projected demand for paediatric isolation facilities (May - October 2014). Occupied bed-days = the cumulative daily occupancy of the 18 isolation beds per month; missed bed-days = duration of hospitalization of patients with pathogens warranting isolation that were not isolated plus days of delay in patients with late implementation of isolation precautions; estimated syndromic bed-day requirement = number of patients with clinical suspicion of viral illness but a negative laboratory test/s for viral pathogens (measured as empiric isolation required from day of admission to a negative test result); projected occupancy = sum of occupied bed-days plus missed bed-days plus estimated syndromic days, minus total bed-days of inappropriate use; Bed-days of inappropriate isolation room use (not shown in figure) = total days per period where isolation rooms were used for inappropriate purposes i.e. where there was no clinical indication for isolation or where a patient was not de-isolated after laboratory testing identified no pathogens or pathogens that did not warrant isolation

isolation room usage and better staff education about indications for isolation. More importantly, at least 135 opportunities for patient isolation were missed (mostly for MDR bacterial and viral infections). Notably, there were few missed isolation episodes for $\mathrm{TB}$, possibly owing to heightened awareness of isolation recommendations for TB among clinicians.

If patients with missed isolation episodes had been appropriately isolated, an overall shortage of 761 isolation bed-days would have been experienced during the study period (i.e. $123 \%$ projected occupancy). However, missed isolation episodes also showed seasonal fluctuation with greatest projected demand over winter (peaking at $208 \%$ or a deficit of 608 bed-days in June 2014). The true need for isolation beds (actual usage, missed episodes and syndromic episodes) is underestimated by the laboratory cross-referencing methodology used, which only identifies children who had appropriate laboratory investigation/s requested and whose specimens had a positive yield.

In contrast to a Canadian study [6] with almost universally correct use of precaution signage and reliable availability of personal protective equipment (PPE), our rates of $71 \%$ and $74 \%$ respectively were lower. Ongoing in-service training of staff and a standardized isolation policy should improve these rates. Encouragingly, alcohol- based handrub was consistently available (89\%), although hand hygiene compliance was lower at $65 \%$. Concurrently collected hand hygiene audit data on the same wards using the "secret shoppers" method, recorded compliance rates of only $41 \%$ (personal communication, Marina Aucamp, Professional Nurse). The Hawthorne effect (where individuals modify or improve behaviour in response to awareness of being observed) may have resulted in our "higher" compliance rate, but these rates are concerning given that staff knew they were handling infectious patients.

Paediatric staff at our institution [18] self-report high rates of adherence $(80 \%)$ to transmission-based precaution recommendations, but observed compliance was low (58 \%). Although appropriate PPE was sometimes unavailable, when it was reliably supplied staff either took no precautions or applied only certain aspects. The additional time and effort required to nurse patients under isolation precautions is well-described [19] and may discourage compliance, together with staff shortages and a lack of education about isolation recommendations.

Limitations of this study include: the short duration (6-months) with inability to estimate annual isolation bed demand and evaluate impact of seasonal disease fluctuations; the tertiary hospital setting (with 18 
isolation beds) which differs from most "open-plan" paediatric wards in low-middle income settings; the observation process (Hawthorne effect) which may have increased staff compliance; the method for identification of missed isolation episodes based on laboratory isolation of specific pathogens, potentially underestimating the true demand for isolation (particularly if syndromic isolation were to be implemented).

Notwithstanding these limitations, this study of paediatric isolation utilization has relevance for our own institution and paediatric wards in other low-resource, TB-endemic settings. To accommodate for peaks in demand and facilitate implementation of syndromic isolation, we propose that new paediatric facilities in our setting have a minimum of $40 \%$ of available beds as single or double cohort isolation rooms. Provision for airborne precautions (needed for half of all isolation episodes in this setting), should be prioritised in new facilities and for renovations of existing paediatric wards in TBendemic settings. Sufficient provision should be made for administrative/office space during design of new wards or facilities, to avoid isolation rooms being inappropriately utilized for non-clinical activities. Syndromic isolation for suspected infection should be implemented (with prompt de-isolation after negative laboratory tests), to reduce risk of infection transmission on children's wards in low-resource settings. To ensure rational and co-ordinated use of isolation beds in our 300-bedded children's hospital, nursing or infection control practitioners could be trained as isolation bed managers. Staff education, written policies and active management of scarce paediatric isolation resources are required.

\section{Conclusion}

Most children admitted to isolation facilities in our TB-endemic setting require airborne precautions. Missed opportunities for patient isolation are common, and could be reduced by implementation of syndromic isolation. Actual and projected demand for isolation facilities exceeds available capacity over the winter season.

\footnotetext{
Abbreviations

CRAB: Carbapenem-resistant Acinetobacter baumanni; DS: Drug-susceptible; DR: Drug-resistant; ESBL: Extended spectrum B-lactamase producing Enterobacteriaceae; HAl: Healthcare-associated infection; HIV: Human immunodeficiency virus; IPC: Infection prevention and control; IQR: Interquartile range; LMIC: Low-middle income country; MDR: Multidrug-resistant; MDR PA: Multidrug-resistant Pseudomonas aeruginosa; MRSA: Methicillin-resistant Staphylococcus aureus; M.tb: Mycobacterium tuberculosis; PMTCT: Prevention of mother to child transmission of HIV; PICU: Paediatric intensive care unit; PPE: Personal protective equipment; TB: Tuberculosis; TCH: Tygerberg Childrens' Hospital.
}

\section{Competing interests}

The authors declare that they have no competing interests.

\section{Authors' contributions}

All authors contributed to study design and critical review of the manuscript. AD carried out the data collection, data cleaning and statistical analysis. All authors read and approved the final manuscript.

\section{Acknowledgements}

The primary author (AD) is supported by the South African Medical Research Council's (MRC) Clinician Researcher Programme and the Discovery Foundation Academic Fellowship (but these funding bodies played no role in the design, data collection, data analysis and interpretation, or writing of the manuscript). The authors wish to thank Mr Hewitt de Jager from the Unit for IPC for assistance with database management, Mr Jean-Marie Vianney Namohoro for assistance with data collection, Mr Darryl Visagie from the hospital IT department, Dr Jean Maritz for access to virology laboratory data and the staff and patients of the Tygerberg Children's Hospital.

\section{Author details}

${ }^{1}$ Department of Paediatrics and Child Health, Division of Paediatric Infectious Diseases, Faculty of Medicine and Health Sciences, Stellenbosch University, PO Box 241, Cape Town 8000, South Africa. ${ }^{2}$ Department of Medical Microbiology, Stellenbosch University and the National Health Laboratory Service (NHLS), Cape Town, South Africa.

Received: 31 July 2015 Accepted: 7 September 2015

Published online: 21 September 2015

\section{References}

1. Siegel JD, Rhinehart E, Jackson M, Chiarello L, the Healthcare Infection Control Practices Advisory Committee. Guideline for Isolation Precautions: Preventing Transmission of Infectious Agents in Healthcare Settings. 2007. Available from: http://www.cdc.gov/hicpac/2007IP/2007isolationPrecautions.html (accessed May 6, 2015).

2. Allegranzi B, Bagheri Nejad S, Combescure C, Graafmans W, Attar H, Donaldson $L$, et al. Burden of endemic health-care-associated infection in developing countries: systematic review and meta-analysis. Lancet. 2011;377(9761):228-41. doi:10.1016/S0140-6736(10)61458-4.

3. Rothe C, Schlaich C, Thompson S. Healthcare-associated infections in sub-Saharan Africa. J Hosp Infect. 2013;85(4):257-67. doi:10.1016/j.jhin.2013.09.008.

4. Kim MH, Mindorff C, Patrick ML, Gold R, Ford-Jones EL, Foster CB, et al. Isolation usage in a pediatric hospital. Infect Control. 1987;8(5):195-9.

5. Foster CB, Sabella C. Health care-associated infections in children. J Am Med Assoc. 2011;305:1480-1.

6. Vayalumkal JV, Streitenberger L, Wray R, Goldman C, Freeman R, Drews S, et al. Survey of isolation practices at a tertiary care pediatric hospital. Am J Infect Control. 2007:35(4):207-11.

7. Langley JM, Hanakowski M, Bortolussi A. Demand for isolation beds in a pediatric hospital. Am J Infect Control. 1994;22(4):207-11.

8. Melendo S, Vilca LM, Albero I, Larrosa N, de Arquer M, Campins M Isolation precautions in a tertiary paediatric hospital. An Pediatr (Barc). 2011;75(1):21-5

9. Sherman GG, Lilian RR, Bhardwaj S, Candy S, Barron P. Laboratory information system data demonstrate successful implementation of the prevention of mother-to-child transmission programme in South Africa. S Afr Med J. 2014;104(3 Suppl 1):235-8.

10. Western Cape Government Provincial Treasury. Municipal Economic Review \& Outlook - Cape Metropolitan Area 2012. Available from: [http://www.westerncape.gov.za/assets/departments/treasury/ dc00_mero_2012_city_of_cape_town_metropolitan.pdf] (accessed May 6, 2015).

11. Magiorakos AP, Srinivasan A, Carey RB, Carmeli Y, Falagas ME, Giske CG, et al. Multidrug-resistant, extensively drug-resistant and pandrug-resistant bacteria: an international expert proposal for interim standard definitions for acquired resistance. Clin Microbiol Infect. 2012;18(3):268-81.

12. World Health Organization. Global Tuberculosis Report 2014. Available from: http://www.who.int/iris/handle/10665/137094 (accessed May 6, 2015).

13. Heyns L, Gie RP, Goussard P, Beyers N, Warren RM, Marais BJ. Nosocomial transmission of Mycobacterium tuberculosis in kangaroo mother care units: a risk in tuberculosis-endemic areas. Acta Paediatr. 2006;95(5):535-9.

14. Schaaf HS, Donald PR, Scott F. Maternal chest radiography as supporting evidence for the diagnosis of tuberculosis in childhood. J Trop Pediatr. 1991;37(5):223-5. 
15. Munoz FM, Ong LT, Seavy D, Medina D, Correa A, Starke JR. Tuberculosis among adult visitors of children with suspected tuberculosis and employees at a children's hospital. Infect Control Hosp Epidemiol. 2002;23(10):568-72.

16. Lee EH, Graham 3rd PL, O'Keefe M, Fuentes L, Saiman L. Nosocomial transmission of Mycobacterium tuberculosis in a children's hospital. Int J Tuberc Lung Dis. 2005;9(6):689-92

17. Weinstein JW, Barrett CR, Baltimore RS, Hierholzer Jr WJ. Nosocomial transmission of tuberculosis from a hospital visitor on a pediatrics ward. Pediatr Infect Dis J. 1995;14(3):232-4.

18. Dramowski A, Whitelaw A, Cotton MF. Healthcare-associated infections in children: knowledge, attitudes and practice of paediatric healthcare providers at Tygerberg Hospital, Cape Town. Paediatr Int Child Health. 2015 May 6. [Epub ahead of print]

19. Saulnier FF, Hubert H, Onimus TM, Beague S, Nseir S, Grandbastien B, et al. Assessing excess nurse work load generated by multiresistant nosocomial bacteria in intensive care. Infect Control Hosp Epidemiol. 2001;22(5):273-8.

\section{Submit your next manuscript to BioMed Central and take full advantage of:}

- Convenient online submission

- Thorough peer review

- No space constraints or color figure charges

- Immediate publication on acceptance

- Inclusion in PubMed, CAS, Scopus and Google Scholar

- Research which is freely available for redistribution 